Voix et Images

voixetimages

\title{
Littérature de circonstances
}

\section{Robert Major}

Volume 15, numéro 1 (43), automne 1989

Jacques Poulin

URI : https://id.erudit.org/iderudit/200821ar

DOI : https://doi.org/10.7202/200821ar

Aller au sommaire du numéro

\section{Éditeur(s)}

Université du Québec à Montréal

\section{ISSN}

0318-9201 (imprimé)

1705-933X (numérique)

Découvrir la revue

\section{Citer cet article}

Major, R. (1989). Littérature de circonstances. Voix et Images, 15(1), 112-116.

https://doi.org/10.7202/200821ar d'utilisation que vous pouvez consulter en ligne.

https://apropos.erudit.org/fr/usagers/politique-dutilisation/ 


\section{Essai}

\section{Littérature de circonstances}

\section{par Robert Major, Université d'Ottawa}

Il y a déjà plus d'un quart de siècle, Gilles Marcotte se faisait traiter de cloporte et de critique dépassé par l'avant-garde du temps, les écrivains de Parti pris. Or, les cloportes ont la vie dure, c'est bien connu; sans doute par effet d'association à la pierre, leur demeure habituelle, ils en prennent la permanence, la solidité et la massive évidence. Gilles Marcotte a enterré ces jeunes Turcs, quand il ne se les est pas associés. Âgé d'à peine quarante ans, il incarnait alors, avec Jean Hamelin et Jean Éthier-Blais, l'institution littéraire québécoise. Il était, avec quelques rares autres, la critique québécoise du temps. À plus forte raison aujourd'hui, parvenu au mitan de la soixantaine, son poids estil réel. Mieux qu'aucun de ses contemporains, il est l'institution. Non seulement parce qu'il dure, se maintient et se renouvelle, première exigence de l'institution, mais aussi parce qu'il incarne les valeurs de celle-ci. Témoin l'importance et la diffusion de ses écrits, les nombreux prix qui l'ont proprement institutionnalisé, et sa présence dans tous les lieux: qu'ils soient populaires (la Presse, l'Actualité), un peu plus élitistes (le Devoir), ou carrément restreints et savants (Liberté et de nombreuses revues universitaires).

Mon propos, en insistant sur le poids et la présence de cet écrivain, n'est pas de momifier Gilles Marcotte: d'ailleurs la vitalité de sa plume m'en empêcherait. Il est de montrer que, étant donné sa propre permanence et sa carrière exemplaire, encore en pleine course: en somme, sa durée, il est peu étonnant que cet essayiste s'intéresse à l'institution littéraire et qu'il en fasse une vibrante apologie. Qu'il nous en faudrait des institutions de ce genre! Et aussi vigoureuses: qu' on nous en donne, nous n'en aurons jamais assez, pour employer la tournure qu'il utilise lui-même (p. 121) en souhaitant des maisons d'édition québécoises sur le modèle Galligrasseuil. Littérature et circonstances ${ }^{1}$ est son douzième volume, je crois, le septième essai (ou recueil d'essais). Et on chercherait en vain de minces plaquettes dans le lot. On voit que le vocabulaire relatif au poids et à la présence massive n'est pas gratuit lorsqu'on parle de cet écrivain.

En effet, je dirais de Gilles Marcotte ce qu'il dit lui-même en parlant d'André Belleau: il est une signature. L'essayiste est l'écrivain d'idées qu'on lit pour sa propre signature, quoi qu'il raconte, quelque domaine qu'il aborde. (p. 311) Mais en changeant l'accent. Quand il parle de Belleau ainsi, c'est pour mettre en valeur sa qualité d'écrivain: Il a mis dans ses essais, même les plus techniques, plus de littérature si je puis dire, plus de travail littéraire, plus de richesses littéraires que la plupart de ses contemporains, poètes ou romanciers, $n$ ' en ont mis dans leurs inventions. (p. 311) Dans le cas de Marcotte, $c$ 'est 
autre chose. Non pas qu'il n'écrive pas bien: au contraire. La même phrase (et, à mon avis, avec plus de justesse...) pourrait être reprise à son compte: il a mis dans ses essais... Mais c'est la qualité des réflexions de Gilles Marcotte, l'intelligence de ses propos, la précision de ses analyses, l'ampleur de sa vision, la richesse de sa culture qui m'intéressent davantage que la qualité de son écriture. Dans la mesure, évidemment, où une telle distinction peut avoir du sens. Ce dernier recueil d'essais est tout à fait à la hauteur de sa réputation.

Littérature et circonstances, donc. Le titre, à prime abord, retient l'attention. Il est des genres littéraires pour lesquels l'association à la «circonstance» est proprement infamante. Le terme est en effet et depuis longtemps chargé de valeurs négatives en littérature. Un poème de circonstance - de Crémazie, par exemple, ou de François Hertel - ne saurait être une véritable poésie, de même qu'une musique de circonstance ne saurait être que banale, prévisible: martiale, peut-être, ou mélodramatique. Quant au roman, dont la "circonstance» se nomme «thèse», on sait en quelle défaveur fut pendant longtemps tenue cette catégorie de récits. L'art véritable, n'est-ce pas, est du côté de l'essentiel, ou de l'éternel.

Curieux cloisonnement, qui a de moins en moins cours, tout en restant vivace. Car on hésiterait avant de dire que tel poème de Miron est un poème de circonstance, même si cela est vrai. Et vrai, non seulement dans l'absolu - toute écriture est entourée, accompagnée, conditionnée par de multiples circonstances qui caractérisent le moment de sa genèse et de son éclosion -, mais vrai aussi dans la conjoncture précise: tel texte, lié à tel événement précis. Mais voilà, on répugne - malgré les efforts des historiens et des sociocritiques - à lier trop étroitement certains genres aux incidences, aux occasions, à la conjoncture et aux contingences: la littérature est ce qui déborde, ce qui ne s'explique pas. Elle doit faire l'illustration, sinon l'éloge, de la gratuité.

Nulle réticence de cet ordre face à l'essai. Il est toujours ce qui s'écrit à partir d'une circonstance, en profitant du prétexte d'une quelconque occurrence. Lukács en faisait d'ailleurs la définition même de l'essai: $a$ côté $d u$ titre de tout essai figure en caractères invisibles: «à l'occasion de...» 2 Sartre, d'ailleurs, ne souhaitait-il pas que ses différents essais ponctuels recueillis dans les dix volumes des Situations soient, de préférence, l'œuvre à lui survivre? Avant les romans, le thêâtre et les grandes œuvres philosophiques. Curieux souhait, mais, à la réflexion, tout à fait pertinent. Car, l'homme mort, qu'y a-t-il de plus apte à le représenter et à le figurer que ces œuvres de circonstances qui manifestent son lien vivant avec l'actualité qui fut la sienne? Ce sont là ses véritables chroniques, l'écriture qui modalisa son existence, l'expression de ses passions ou de ses intérêts. Dans son étude fondamentale du genre (Voix \& images, hiver 1983), Robert Vigneault demande:

Qu'est-ce qu' un essayiste, en effet, sinon un artiste de la vie, celui qui imprime à l'expérience vécue la forme de ses questions? [...] Exigeante quête de la substance, l' essai prend forme en soumettant la contingence du vécu à la haute exemplarité des idées (valeurs). (p. 321-322) 
On aura remarqué le vocabulaire: la vie, l'expérience, la contingence, le vécu... La contingence du vécu, à interroger et à mettre en forme. Mais, le plus souvent, ce vécu a d'abord été médiatisé par une œuvre quelconque, littéraire ou autre, et l'essayiste est aussi critique, au sens le plus noble du mot. La «circonstance» est alors double, ou bi-polaire: vécu immédiat, d'une part, œuvre qui rédime ce donné brut (Vigneault), d'autre part. L'essai est donc doublement lié à la circonstance, dont il peut - unique parmi les genres littéraires - se réclamer, sans pudeur, sans honte et sans faux-fuyants.

En choisissant son titre, Gilles Marcotte le savait bien. Avec son pluriel, surtout, le terme est un étendard, l'affirmation d'une position et d'une pratique littéraires. Position et pratique élucidées dès les premières phrases de l'introduction. L'auteur refuse sans ambiguîté toute conception désincarnée de la littérature: la littérature n'est pas expression pure de la subjectivité, ou encore collection de beaux objets sans rapport avec la vie commune, avec ce que nous appelons l' histoire (p. 9). Le texte littéraire est compromis, c'est-à-dire exposé, impliqué, mêlé à la vie, jouant de risque. Sa relation avec le réel et avec la vie commune, ses liens intimes avec nos façons de penser la vie et la position que nous y occupons - c'est-à-dire, les idéologies -, sont pour l'essayiste une donnée première ${ }^{3}$. Mais, simultanément, tout en déboulonnant la littérature de son piédestal, tout en la basculant dans la rue, Gilles Marcotte lui assure un statut privilégié. La littérature n'est pas simple reflet ou expression de la vie commune: elle constitue une pratique particulière qui nous permet de voir et de comprendre ce que nous vivons. À l'instar des mathématiques, discours logique des sciences, fiction et abstraction qui les rend pensables, la littérature abstrait, dégage les lignes de force, rend pensable l'actualité et l'histoire. En somme, elle est l'herméneutique du réel.

Mais encore là, comme pour s'interdire toute valorisation excessive de la littérature, à ces phrases de l'Introduction, Gilles Marcotte posera en contrepoids le très bel essai final, "Qui a peur du pygargue roux?», réflexion subtile et discrètement angoissée sur cet irrépressible besoin d'écrire. L'étonnant, c'est $q u$ 'on écrive encore (p. 313), constatera-t-il, après tant de siècles où tout a été dit et redit. L'écrivain est-il autre chose qu' un névrosé qui s'ignore? (p. 314) L'écriture, consciente de son absurdité, n'est-elle pas devenue que critique d'elle-même, l'arme de sa propre disparition (p. 318), ou encore, entreprise sans finalité, définie par le gaspillage et la dilapidation? Face à la sursaturation de l'écrit, exerçant une action dont le caractère tautologique ne peut lui échapper, l'écrivain est devenu, littéralement, celui qui ne veut rien savoir (p. 325) et qui est condamné, depuis un siècle, névrotiquement, à écrire ex cathedra ignorantiae (p. 329). Mais alors, à quoi rime la littérature? Évanouies, ces hautes fonctions qu'on est porté à lui attribuer. Peut-être n'écriton que par amitié? Peut-être la littérature n'est-elle que l'affirmation d'une possibilité de rencontre et soif de l'autre, banale et poignante tentative d'unir deux solitudes dans l'espace d' un mot nouveau (p. 331)?

On le voit: s'ouvrant sur une position de principe et sur un certain nombre d'affirmations posées avec assurance, le recueil se termine sur des interrogations 
et sur une sourde inquiétude. Incertitude, hésitation, recherche indécise, conaturelles à l'essai. Ainsi, ces articles et études écrits au cours des quinze dernières années (p. 9) ${ }^{4}$, c'est-à-dire liés à des circonstances particulières, inséparables du moment, forment tout de même un recueil, un ensemble. Un recueil qui se veut articulé autour de deux axes distincts: une première section, les deux tiers du volume, où la littérature québécoise est saisie dans ses relations avec l'Histoire, l'institution, les idéologies; une deuxième, où le critique ne veut qu'accompagner certains écrivains (dont, cette fois, quelques poètes français) dans leur aventure de l'écriture. En vérité, cette division en sections est purement volontaire et plutôt spécieuse. Je ne crois pas que Gilles Marcotte y aille autrement dans la deuxième section; s'il entre par une autre porte (p. 11), c'est pour se retrouver dans la même pièce et y tenir essentiellement des propos de même nature. Ses intérêts, eux, n'ont pas changé. Chacun des essais de la deuxième section, consacrés soit à Ferron, Brault, Jasmin, Crémazie... pourrait tout aussi bien se retrouver coiffé du titre de la première section ${ }^{5}$. Si je le signale, ce n'est pas en guise de reproche. C'est au contraire pour affirmer l'unité de ton du recueil, la constance des préoccupations de l'essayiste. D'ailleurs, c'est bien connu que les essayistes ont des problèmes avec leurs tables des matières, le plus souvent arbitraires et déroutantes, et qu'on aurait tort de s'y attarder.

Ce sont les essais individuels qui doivent nous retenir. Certes, il n'y a là rien de neuf au sens strict, puisque tout a été publié auparavant. Mais relire, c'est le commencement de la lecture (p. 122), ainsi que le dit Marcotte lui-même en parlant des poètes de l'Hexagone. L'occasion nous est ainsi donnée de «commencen notre lecture de toutes sortes d'aspects de la littérature québécoise. Même quand le sujet nous est bien connu (souvent grâce à d'autres écrits de Marcotte), la vigueur de la pensée et la finesse des rapprochements provoquent des redécouvertes ou des remises en question. Et chacun, en fonction de ses intérêts, de ses ignorances ou de ses lacunes, fera ici bombance. Signaler l'un ou l'autre essai n'a qu'un intérêt anecdotique puisque chacun sera attiré par un aspect différent; mais donnons tout de même, en terminant, quelques exemples rapides. Ainsi, les trois essais sur l'Hexagone m'ont laissé quelque peu indifférent (effet de saturation, peut-être), mais ceux sur les années trente (la Relève et ses polémiques) m'ont passionné; je crois que la perception de l'américanité dans nos lettres est assez courte et que Gilles Marcotte n'a peut-être pas suffisamment écouté quand nos écrivains lui ont conté l'Amérique 6 , mais par ailleurs, sa vision de la religion comme voie d'accès à l'universel pour notre littérature m'a semblé d'une profonde vérité; j'ai trouvé l'essayiste particulièrement sévère à l'égard de Jacques Ferron (même si l'analyse est magistrale), trop louangeur à l'égard de Belleau (chacun est présentement en train d'ajouter sa pierre à l'édifice Belleau et cette entreprise de monumentalisation - qu'on me passe le mot! - menée par ses connaissances laisse plutôt songeur), très juste dans son appréciation du premier Jasmin; je crois qu'il serait temps que Gilles Marcotte revoie son évaluation de Gérin-Lajoie, mais je dois me dire profondément d'accord avec sa critique de Poupart et consorts; finalement, j'ai savouré la fine ironie du premier essai («Institutions et courants d'air») et je ne saurais dire tout le bien que je pense du dernier («Qui a peur du pygargue roux?»). 
Voilà donc pour un bref échantillonnage de mes intérêts et lacunes, sans pertinence pour un autre lecteur. À chacun de lire. Ces essais intelligents et subtils, savants mais dénués de jargon, offrent un plaisir constant à l'esprit.

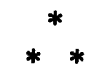

Le sociologue est romancier; l' ethnologue est conteur, dira Gilles Marcotte à un moment donné (p. 251) pour cerner l'identité de l'œuvre de Ferron. Je retiens de la sentence, non pas la catégorisation des genres narratifs, mais son implicite: le passage sans heurts d'une discipline dite scientifique à la littérature. On l'admettra d'autant plus volontiers qu'ici, au pays, les sociologues se sont souvent intéressés à la production littéraire et que, d'autre part, quelquesuns parmi eux sont parmi nos meilleurs écrivains. J'en veux comme illustration supplémentaire et récente les demiers livres de Marcel Rioux et de Guy Rocher. Marcel Rioux qui vide ses fonds de tiroir, en quelque sorte, et nous offre ses Anecdotes saugrenues ${ }^{7}$, petites aventures et historiettes, fruits pour la plupart d'une longue expérience d'enquêtes sur le terrain: un livre savoureux, sans prétention, regorgeant d'humour et de sagesse. Guy Rocher, quant à lui, qui jusqu'à présent s'était résolument cantonné à l'intérieur de sa discipline et qui accepte cette fois, sollicité par les questions habiles et intelligentes de Georges Khal, de faire le bilan de sa vie et de sa génération. Entre les rêves et l'histoire 8: voici un livre qui servira d'inspiration à tous les apprentis sociologues ou intellectuels en herbe; et pour nous, littéraires, une série d'entretiens qui se lit comme un roman.

1 Gilles Marcotte, Littérature et circonstances, Montréal, l'Hexagone, 1989, 352 p. (Essais littéraires).

2 Georges Lukács, I'Âme et les formes, Paris, Gallimard, 1974, p. 29-30 (Bibliothèque de philosophie).

3 Plus loin, l'affirmation sera encore plus péremptoire: je pose que toute cuvre littéraire [...] parle de la société, fait parler la société, ou que la société parle en elle (p. 153).

4 En réalité, il faudrait allonger la période. L'étude du thème du pays dans la poésie de l'Hexagone est de 1969, comme le signale Gilles Marcotte lui-même dans sa note, p. 338.

5 Par exemple, Ferron y est étudié comme ethnologue du Québec, l'œuvre de Jacques Brault est dite un des sismographes les plus exacts de l'histoire du Québec au cours du dernier quart de siècle (p. 272), le premier roman de Jasmin est étudié comme annonçant ce qu'allait devenir le roman québécois d'aujourd' hui (p. 241), Crémazie est établi comme le seul véritable lecteur de son siècle (p. 227), et ainsi de suite.

6 D'ailleurs, les affirmations du court essai «Découvrir l'Amérique» sont ellesmêmes sévèrement nuancées par l'essai sur Robert Charbonneau et notre signification américaine.

7 Marcel Rioux, Anecdotes saugrenues. Historiettes, Montréal, l'Hexagone, 1989, $119 \mathrm{p}$.

8 Guy Rocher, Entre les rêves et l'histoire. Entretiens avec Georges Khal, Montréal, VLB éditeur, 1989, 232 p. (Études québécoises). 